\title{
Duplas Psicosociales. Demandas y desafíos desde la visión de los profesionales que intervienen en las escuelas de la Región de Ñuble
}

Psychosocial Pair. Demands and Challenges from the Perspective of the Professionals Involved in the Schools of the Nuble Region

\author{
Héctor Cárcamo-Vásquez \\ Universidad del Bío-Bío, Chile \\ ORCID: https://orcid.org/0000-0003-2045-343X \\ Carmen Gloria Jarpa-Arriagada* \\ Universidad del Bío-Bío, Chile \\ ORCID: https://orcid.org/0000-0002-9896-5649 \\ María Teresa Castañeda-Díaz \\ Universidad del Bío-Bío, Chile \\ ORCID: http://orcid.org/0000-0001-7594-9630
}

Recibido 17-05-19 Revisado 30-08-19 Aprobado 26-11-19 En línea 02-12-19

*Correspondencia

Email: cjarpa@ubiobio.cl
Citar como:

Cárcamo-Vásquez, H., Jarpa-Arriagada, C., \& CastañedaDíaz, M. (2020). Duplas Psicosociales. Demandas y desafíos desde la visión de los profesionales que intervienen en las escuelas de la Región de Ñuble, 8(2), e324. Doi: http://dx.doi.org/10.20511/pyr2020.v8n2.324 


\section{Resumen}

El objeto de estudio que se aborda en este artículo corresponde a desafíos y demandas concretas que identifican los profesionales que conforman las duplas psicosociales de diversas escuelas de la Región de Nuble. Dada la naturaleza del objeto, el abordaje corresponde a la metodología cualitativa a partir del enfoque sociofenomenológico. La producción de datos se realizó a partir de 10 grupos de trabajo constituidos por profesionales que conforman duplas psicosociales. Como principales hallazgos encontramos que, desde la perspectiva de los agentes, el quehacer de las duplas psicosociales está mediado por tres nudos críticos fundamentales, los cuales operan desde una lógica de totalidad: contexto, normatividad y acción. De esta forma, las duplas psicosociales se instalan en las escuelas como consecuencia de un entramado de políticas pro-inclusión, que presiona hace más de una década. Su inserción en el sistema educativo presenta diversas dificultades ligadas a la precariedad laboral, funciones y roles difusos, así como inestabilidad y discontinuidad de los procesos de intervención psicosocial con niños y niñas y sus respectivas familias. En suma, se observa una clausura disciplinar de corte pedagógico que obstaculiza la integración de la dupla psicosocial como dispositivo validado para la mejora escolar.

Palabras clave: Política Social; Sistema educativo; Escuela desfavorecida; Subvención educativa, Desigualdad social.

\section{Summary}

The object of study that is addressed in this article corresponds to specific challenges and demands that professionals that make up the psychosocial pairs of several schools in the Nuble Region identify. Due to the nature of the object, the approach corresponds to the qualitative methodology based on the socio-phenomenological approach. The data production was made in 10 working groups constituted by professionals that make up psychosocial pairs. As main findings we see that, from the perspective of the actors, the task of the psychosocial pairs is mediated by three fundamental critical issues, which operate from totality: context, normativity and action. Thus, psychosocial pairs work in schools as a result of a pro-inclusion policies framework, which has been pressing for more than a decade. Its incorporation to the educational system presents various difficulties linked to job insecurity, diffuse roles and functions, as well as instability and discontinuity of psychosocial intervention processes with children and their respective families. In sum, there is a pedagogical disciplinary closure that hinders the integration of the psychosocial pair as a validated unit for school improvement.

Keywords: Social Policy; Educational System; Disadvantaged School; Educational Subsidy; Social Inequality

\section{Introducción}

Para nadie es sorpresa reconocer que Chile, a partir de mediados de 1970 con la puesta en marcha de los ajustes estructurales dirigidos a impulsar la implementación del modelo neoliberal, inclinó la balanza hacia lo privado, reduciendo el Estado en términos de funciones e injerencias en el devenir de la sociedad chilena, situación que poco a poco fue transformando a Chile en uno de los países más desiguales del mundo (PNUD 2017, French-Davis 1999, Salazar \& Pinto 1999). En este contexto, la educación -uno de los ejes estratégicos para el desarrollo de los países- no quedó ajena a estas transformaciones. Un claro ejemplo de ello se aprecia en el proceso de privatización y municipalización de los establecimientos educacionales de todo el país y la consecuente segregación del estudiantado por situación socioeconómica y lugar de residencia.

Tal como sostienen Torche y Wormald (2004), en el marco de las sociedades modernas se da "una fuerte expansión de la participación de las personas en el sistema educacional, en tanto 
que los logros educacionales han adquirido una creciente importancia en la obtención de empleo en el mercado de trabajo" (Torche \& Wormald 2004, p. 29). Precisamente, sobre la base de lo expuesto por estos autores, podemos afirmar que, en torno a la educación se configura un discurso que la posiciona como un potente motor de movilidad social ascendente, expandiéndose la oferta y las oportunidades de acceso a la educación en todos sus niveles. Tras largos años de andar, las consecuencias del modelo se hacen sentir con fuerza en la educación formal. Esa expansión y ampliación de oportunidades comienza a cuestionarse. Los resultados que arrojan los instrumentos estandarizados a nivel nacional e internacional respecto del desempeño académico de los y las estudiantes adscritos al sistema educacional chileno, tales como el SIMCE y la prueba PISA, ponen de relieve una profunda brecha entre quienes acceden a establecimientos educacionales particulares pagados, particulares subvencionados y municipalizados.

A este respecto, Bellei (2013) plantea que la forma de operar del sistema educacional chileno va modelando una articulación irrevocable entre la segregación socioeconómica y la segregación académica, en tanto factores de inequidad educativa. De este modo puede afirmarse que "los efectos de la segregación se producen tanto directamente sobre los alumnos y sus procesos de enseñanza-aprendizaje, como indirectamente a través del ambiente escolar y los profesores" (Bellei 2013, p.339).

El reconocimiento de esta profunda inequidad educativa que se consolidó en Chile durante largas décadas lleva al Estado de Chile a impulsar diversos esfuerzos orientados a mejorar la calidad de la educación, disminuir la inequidad y desigualdad social producto de la profunda brecha cimentada por el mercado educativo. Al tenor de esta situación, Raczynski y Muñoz (2007) afirman que uno de los desafíos que debía afrontar el sistema educacional chileno era favorecer una mayor equidad a partir de la corrección del sistema de financiamiento de las escuelas chilenas, considerando como elemento fundamental para el análisis los niveles de vulnerabilidad de la población a la que atienden. A este respecto Raczynski, Muñoz, Weinstein y Pascual (2013) sostienen que uno de los esfuerzos notables y trascendentales para hacer frente a esta problemática fue la aprobación de la Ley de Subvención Escolar Preferencial, SEP (Ley 20.248 de 2008). En términos esenciales, la Ley 20.248, opera sobre la base de una lógica redistributiva dirigida a mejorar la equidad al interior del sistema escolar chileno (Saracostti 2013). En palabras de Raczynski, et al. (2013)

"la SEP tiene el propósito de mejorar los niveles de equidad del sistema escolar, entregando mayores recursos a las escuelas que atienden a esos niños. El Estado entrega esta nueva subvención a aproximadamente un tercio del alumnado, corrigiendo, al menos, en parte, las desigualdades de origen" (p. 166).

Cabe consignar que la puesta en marcha de la Ley SEP se forja sobre la base de una lógica diferenciadora con respecto de las formas en que se venían desarrollando las políticas educativas en Chile. La ley SEP pretende romper con la lógica exógena descendente clásica otorgando protagonismo a los propios establecimientos educacionales quienes, junto a sus respectivos sostenedores, deben propender hacia el diseño de planes de trabajo debidamente situados desde los contextos específicos en los cuales se desean implementar.

Asumiendo el aprendizaje como un proceso multidimensional, es necesario reconocer que son diversos los factores que intervienen en él, muchos de los cuales trascienden la esfera del cuerpo docente, por ejemplo, la implicación de las familias en el proceso educativo, el contexto sociocultural en el que se encuentra situada la escuela y la autoestima de los y las estudiantes, por mencionar solo tres de los factores más reportados por la literatura especializada (Gubbins 2016, Garreta 2015, Ferrada 2012, entre otros). En el marco de la ley SEP se destinan recursos orientados a contratar profesionales que apoyen la labor docente en aquellas dimensiones que trascienden el quehacer del profesorado pero que, no obstante, aquello, inciden en el proceso de enseñanza y aprendizaje. En este marco se insertan las duplas psicosociales. 
En palabras de Gatica (2015)

“Al contar los colegios con un equipo profesional exclusivo para la intervención psicosocial, se mejoran las condiciones de concretar un trabajo de calidad en el aprendizaje de los estudiantes, atendiendo a los requerimientos que el establecimiento demanda, levantando planes coherentes, con sus propias necesidades y delineando estrategias acordes al contexto social y psicológico de los estudiantes y la comunidad escolar" (Gatica 2015, p. 5).

\section{Las duplas psicosociales en el contexto de la escuela}

Entenderemos por dupla psicosocial al equipo profesional conformado por un trabajador social y un psicólogo que prestan apoyo psicológico y asistencia social a los estudiantes y sus familias en los establecimientos educacionales que presentan altos niveles de vulnerabilidad, medida a través del índice de vulnerabilidad escolar (IVE) de la Junta Nacional de Auxilio Escolar y Becas (2005).

En este contexto, la intervención de duplas psicosociales en el ámbito escolar se fundamenta en una concepción del sistema educacional como fenómeno social complejo, que plantea la necesidad de abordar la educación desde un enfoque interdisciplinario (Chacón 2007). Dicha complejidad incluye la multidimensionalidad de los fenómenos educativos, la naturaleza compleja de la enseñanza, la importancia de los contextos socio-culturales y la perspectiva situada y distribuida de la cognición (Korthagen, Loughran \& Russell 2006; Lave \& Wenger 1991).

Desde la perspectiva compleja descrita, el desafío de las políticas educativas apunta al aseguramiento de una educación de calidad e integración social en Chile, considerando la multiplicidad de factores involucrados en la educación (Gatica 2016). En este contexto se enmarcan la Ley de Subvención Educacional Preferencial (2008); el Ajuste al Marco Curricular de la Enseñanza Básica y Media (2009); la Ley General de Educación (2009); la Ley sobre Agencia de Calidad (2011); el Sistema de Aseguramiento de la Educación Básica y Media (2011); la creación de la Superintendencia de Educación (2012); las Bases curriculares de Educación Básica (2012), en las que se advierte un foco interdisciplinario tanto en su diseño como en su implementación.

Si se mira la educación desde una óptica compleja, es necesario relevar aspectos distintos de los netamente educativos como son el aula, los materiales escolares, las horas pedagógicas, los recursos tecnológicos. En nuestro país, las desigualdades económicas y sociales impactan negativamente sobre la calidad de la educación. La segregación social de las escuelas perjudica a quiénes poseen menos recursos materiales y culturales, excluyéndoles de las condiciones que favorecen una mejor calidad de la educación como son docentes calificados, buena convivencia escolar, mayor acceso a oportunidades de aprendizaje (Bellei 2013, Valenzuela, Bellei \& De Los Ríos 2010). En este marco, las intervenciones psicosociales en Chile se desarrollan en las escuelas que presentan altos índices de vulnerabilidad, catalogados como escenarios educativos complejos donde los factores psicosociales (desarrollo cognitivo, del lenguaje y socioemocional) tienen alta incidencia en la vulnerabilidad de los estudiantes. Avalan esta implementación, diversos estudios que concluyen sobre su contribución al aprendizaje y al desarrollo de los alumnos y sus familias (Gatica 2015; Yeager \& Walton 2011).

\section{Modelos de intervención individuo-familia en la escuela}

Para aproximarnos a los modelos de intervención individual-familiar aplicados en las escuelas chilenas, desagregaremos el análisis en tres dimensiones: (i) la dimensión de formación profesional de trabajadores sociales y psicólogos que operan bajo el rótulo de "dupla psicosocial"; (ii) la dimensión político-normativa de la nueva institucionalidad escolar y (iii) la tensión entre inclusión-exclusión que satura el nuevo management público (NMP) y la intervención psicosocial en las escuelas. 
La formación de Trabajadores Sociales y Psicólogos tiene importancia ya que resulta inevitable que los profesionales llamados a "intervenir" a niños, niñas, adolescentes y familias, actuarán en la realidad según sus operaciones de distinción (Maturana 1996), fuertemente influidas por la formación recibida. En efecto, podemos afirmar que en Chile se ha instalado con fuerza el paradigma del "Modelo Centrado en las Competencias", que ha impactado en la formación del Trabajador Social y del Psicólogo, pero no necesariamente hacia la renovación de modelos de intervención psicosocial, sino más bien hacia el predominio de la tensión entre Modelos psicodinámicos y Modelos Sistémicos.

Por una parte, los modelos psicodinámicos, de tradición psicoanalítica, expresan una configuración patologizante del "paciente" o "usuario", centrado en sus hándicaps y debilidades, proponiendo intervenciones dirigidas a "normalizar" la situación, atribuyendo a los profesionales el rol de expertos. Por otra parte, los modelos sistémicos, operan bajo una lógica de construcción social, dónde el individuo o familia a intervenir adquiere un rol protagónico, desde sus potencialidades y recursos personales, donde la configuración de una relación profesional simétrica, colaborativa y emancipadora resulta un componente imprescindible para la actuación profesional (Du Ranquet 1996).

En este contexto, el quehacer del psicólogo parece seguir gobernado por acciones de evaluación y diagnóstico, diseño, ejecución y evaluación (González, González y Vicencio 2014), de fuerte tradición psicodinámica y de concepción clínica de la intervención. Asimismo, la práctica psicosocial de trabajadores/as sociales y psicólogos está fuertemente influenciada por el tipo de vínculo entre experto y usuario, modelado por los requerimientos de la "institución" en términos de normalizar la situación y lograr la adaptación del sujeto a la sociedad (Gatica \& Hess 2011), todo lo que se inclina fuertemente a la hegemonía del modelo psicodinámico.

Respecto de la dimensión político-normativa de la nueva institucionalidad escolar, podemos mencionar la importancia de leyes y normativas recientes que han impactado en la nueva estructura de las escuelas, a saber, Política Nacional de Educación Especial (MINEDUC, 2005), Decreto 170 (MINEDUC, 2009), Ley 20.248 de Subvención Escolar Preferencial (MINEDUC, 2008), Política de convivencia escolar (MINEDUC, 2011), Programa de educación en sexualidad, afectividad y género (MINEDUC, 2012), Ley 20.609 de no discriminación (SEGEGOB, 2012), Proyecto de Ley de migración y extranjería (Gobierno de Chile 2013) y Ley 20.845 sobre la Inclusión Escolar (2015). Sobre el particular, este entramado de recientes normas ha contribuido a forjar un nuevo discurso centrado en ideas de inclusión, igualdad y perspectiva de derecho, pero, a su vez, revela una evidente lógica de nuevo management público (Infante \& Matus 2009; Matus e Infante 2011).

En este sentido, Apablaza (2014, 2016, 2017), desarrolla de manera consistente una aproximación a la escuela como dispositivo de gobierno, donde predomina la racionalidad instrumental heredada del positivismo, con las inmediatas consecuencias de modos de subjetivación que no logran hacerse cargo del nuevo régimen de poder centrado en la inclusión, la igualdad y la perspectiva de derecho, generando serias limitaciones para la aceptación de las diferencias. Apablaza (2017b) identifica tres nudos en las escuelas en lo que respecta a intervención psicosocial: (i) La política de convivencia escolar, específicamente el abordaje de la violencia; (ii) Las Necesidades Educativas Especiales y el cumplimiento de la "Inclusión" y (iii) las brechas de Género. En efecto, los resultados de sus investigaciones se inclinan a mostrar que, no obstante, disponer de una abundante normativa pro-inclusión, los problemas psicosociales en las escuelas se siguen trabajando con modelos psicodinámicos de intervención, lo que ella denomina Tecnologías "Psi" (Apablaza 2017a).

Respecto de la tensión entre inclusión/exclusión en el nuevo management público (NMP) y la intervención psicosocial en las escuelas, nos situaremos desde el fenómeno de la inclusiónexcluyente (Ezcurra 2011). En primer término, entendemos la exclusión como un proceso de acumulación histórica, opuesto a la noción de un simple acontecimiento. Así, no cabe hablar de 
estudiantes en riesgo de exclusión, sino de condiciones sociales y educativas que representan un contexto de riesgo y de vulnerabilidad (Muñoz, González y Domínguez 2009). Por consiguiente, cuando una escuela excluye, este proceso representa un difuso recorrido entre la implementación de políticas de inclusión y la exclusión como una manifestación multifactorial de la reproducción social asociada a diferencias socioeconómicas, de capital cultural (Giraldo-Zuluaga 2015), y a componentes de carácter institucional. Estos últimos, estarían dados por la lógica predominante de racionalidad instrumental, coherente con el NMP.

Una de las manifestaciones "psicosociales" de la inclusión-excluyente ocurre cuando la dupla psicosocial opera desde las Tecnologías "Psi" (Apablaza 2017a). Bajo este paradigma, los profesionales actúan desde un saber experto, sobrepatologizando a las personas y a las familias y actuando desde un dispositivo metodológico que implica estudiar, analizar, pesquisar e intervenir, reproduciendo la lógica de "Objetos de estudio" más que de "actores sociales". En definitiva, una actuación profesional dirigida a familias y niños, niñas y adolescentes, incluye en tanto son beneficiarios de un plan de acción, pero excluye ya que estigmatiza, patologiza y etiqueta en categorías como "pobre", "incompetente", "disruptivo", "disfuncional", "violento", "integrado", entre otros.

\section{Método}

El objeto de estudio que se aborda en este artículo corresponde a desafíos y demandas concretas que identifican los profesionales que conforman las duplas psicosociales de diversas escuelas de la Región de Nuble. Dada la naturaleza del objeto que se desarrolla en este artículo, el camino metodológico para su abordaje corresponde a la metodología cualitativa a partir del enfoque sociofenomenológico; entendiendo que el fenómeno educativo -en el más amplio sentido de la palabra- no solo se corresponde con un problema de investigación, sino que también como un espacio de lucha política (Bourdieu \& Passeron 1979, Rockwell 2009).

Se trabaja con los discursos construidos por profesionales que conforman las duplas psicosociales (trabajadores sociales y psicólogos) y profesores de integración que apoyan el trabajo en aula de diversas escuelas de la Región de Ñuble. Se trabaja con un total de 78 sujetos de los cuales 15 corresponden a hombres y 63 a mujeres. Los sujetos son distribuidos en 10 grupos de trabajo. En estos grupos se abordan requerimientos específicos que reconocen necesarios de cubrir y alternativas de solución visualizadas por los propios agentes. La información proporcionada por cada grupo de trabajo se sistematiza y consolida en un documento dispuesto para el análisis. De este modo, el material es transcrito y luego categorizado siguiendo los principios del paradigma de la codificación (Trinidad, Carrero y Soriano 2006). Tras este proceso se procede a realizar un análisis combinando la estrategia vertical (grupo por grupo) y la temática, con el fin de identificar elementos constitutivos de regularidad y dispersión de los discursos. Las categorías corresponden a las siguientes:

- Condiciones laborales.

- Delimitación del quehacer de la dupla psicosocial.

- Intervención de la dupla psicosocial.

\section{Resultados}

Los resultados que a continuación se exponen centran la atención en las tres categorías centrales de carácter selectivo siguiendo los principios del paradigma de la codificación (Trinidad, Carrero y Soriano 2006): condiciones laborales, delimitación del quehacer de las duplas psicosociales, intervención de la dupla psicosocial. Cada uno de los apartados representa un nudo crítico específico identificado por los grupos de trabajo, la presentación de resultados permitirá reconocer las dificultades y las demandas específicas que consideran necesarias de cubrir para afrontarlas de forma exitosa. 


\section{Condiciones laborales}

Tras la codificación del material producido por los grupos de trabajo, uno de los aspectos que emerge como fundamental en el quehacer de los profesionales que constituyen las duplas psicosociales, dice relación con las condiciones laborales. A este respecto, cabe consignar que se aprecia una regularidad discursiva que permite agrupar cuatro elementos constitutivos del nudo crítico sobre la base de cuatro dimensiones: carga horaria, espacio físico, distribución de recursos y recursos humanos (ver figura 1).

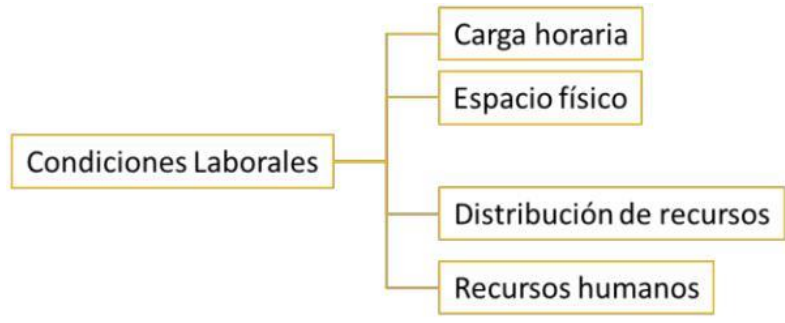

Figura 1. Dimensiones constitutivas del nudo crítico: condiciones laborales.

\section{Carga Horaria}

Una de las dimensiones constitutivas del nudo crítico identificado por los sujetos participantes, dice relación con la carga horaria. A este respecto, se aprecia una clara regularidad discursiva intergrupos. Cuando refieren a la carga horaria, es posible reconocer dos elementos fundamentales que la configuran como una dimensión a considerar. El primero de estos elementos se corresponde con un aspecto de carácter gremial, por cuanto se alude a la inestabilidad laboral con modelos de contratación de variada duración. El otro elemento refiere directamente al ámbito técnico profesional, pues, la inestabilidad laboral se aprecia en la alta rotación de los profesionales y la consecuente discontinuidad de las intervenciones. A la luz de lo informado por los sujetos, este aspecto atenta contra una adecuada atención psicosocial de los agentes que la requieren, poniendo en jaque uno de los principios rectores de la ley SEP: mejorar los niveles de equidad en el sistema escolar.

Los elementos expuestos, se ven reflejados con claridad en el siguiente fragmento:

"La inestabilidad de carga horaria y permanencia dentro del mismo establecimiento educacional, considerando las contrataciones semestrales y anuales que existen actualmente, influyendo en la continuidad de las intervenciones psicosociales que ameritan los alumnos y en ocasiones su grupo familiar y esto no facilita los procesos de reparación que pueda requerir un estudiante". (Grupo de Trabajo N²)

\section{Espacio Físico}

Otra de las dimensiones constitutivas del nudo crítico condiciones laborales, corresponde al espacio físico. De acuerdo a lo reportado por los grupos de trabajo, el espacio físico se configura como una dimensión fundamental en el quehacer de los profesionales que conforman las duplas psicosociales, puesto que, al tratarse de casos en su mayoría de mediana y alta complejidad, es crucial disponer de espacios físicos privados y debidamente equipados para realizar entrevistas, tanto con familias como con niños y niñas, así como las respectivas sesiones de intervención psicosocial. La inexistencia de espacios adecuados en muchos establecimientos educacionales dificulta gravemente el cometido de las duplas psicosociales. De este modo, las condiciones físicas - existentes e inexistentes-ponen de manifiesto la precariedad en que los agentes deben llevar a cabo su ejercicio profesional. 
A este respecto los integrantes del grupo $\mathrm{N}^{\circ} 3$ destacan lo siguiente:

"Falta de espacios físicos en los establecimientos que no nos permite realizar una intervención privada" (Grupo de Trabajo N³).

\section{Distribución de Recursos}

Como tercera dimensión constitutiva del nudo crítico condiciones laborales, refieren al uso de los recursos económicos que cada establecimiento educacional percibe a través de los instrumentos vigentes para la atención de niños y niñas con mayor nivel de vulnerabilidad social. A pesar de existir diferentes instrumentos, tales como la ley SEP ( $\left.\mathrm{N}^{\circ} 20.248\right)$, la ley de inclusión escolar $\left(\mathrm{N}^{\circ} 20.845\right)$, los sujetos participantes sostienen que no siempre los recursos son debidamente focalizados por los equipos directivos de los establecimientos educacionales.

A este respecto el grupo de trabajo $\mathrm{N}^{\circ} 3$ reporta lo que sigue:

"Distribución de recursos que no llegan a las reales necesidades". (Grupo de Trabajo N³).

El criterio técnico no siempre es el priorizado por parte de los responsables de distribuir y asignar los recursos. De acuerdo a lo debatido durante la plenaria, se puede desprender que en muchas ocasiones el criterio técnico queda subyugado a la contingencia.

\section{Recursos Humanos}

Finalmente, la otra dimensión a partir de la cual se configura el nudo crítico condiciones laborales dice relación con el recurso humano. Tal como indicásemos en la dimensión carga horaria, esta dimensión es altamente relevante por cuanto advertimos que, los reportes proporcionados por los grupos de trabajo confluyen en dos aspectos específicos. El primer aspecto centra la mirada de los agentes respecto a la falta de profesionales para atender adecuadamente a niños, niñas y sus familias.

A este respecto el grupo $\mathrm{N}^{\circ} 10$ nos indica lo siguiente:

"Falta de recursos humanos. Más profesionales de otras áreas" (Grupo de Trabajo $\left.\mathrm{N}^{\circ} 10\right)$.

En este sentido, tanto el número de profesionales y las horas contratadas para dar cumplimiento a las funciones de las duplas psicosociales son consideradas insuficientes, situación que se da con independencia del tipo de administración del establecimiento educacional. Tal como nos lo indica el grupo de trabajo $\mathrm{N}^{\circ} 4$ :

"Déficit de horas para trabajadores sociales en colegios subvencionados" (grupo de Trabajo $\mathrm{N}^{\circ} 4$ )

Tanto el número de profesionales como la modalidad de las contrataciones tienen un efecto directo sobre la rotación de profesionales. En palabras de los integrantes del grupo de trabajo $\mathrm{N}^{\circ} 10$, se constata en los diferentes establecimientos educacionales una:

"Alta rotación de profesionales, falta de continuidad" (Grupo de Trabajo $\left.\mathrm{N}^{\circ} 10\right)$

Situación que tiene un efecto directo en la calidad de los procesos de intervención de mayor complejidad, tal como se verá en el punto 5.3.

\section{Delimitación del quehacer de las duplas psicosociales}

Esta categoría da cuenta de cómo las duplas psicosociales relatan su especificidad profesional en el contexto de un entramado variado y, muchas veces inconexo, de normativas y reglamentaciones que enuncian muy someramente su función. Por consiguiente, este nudo crítico se expresa en dos 
dimensiones: (i) la poca claridad de los roles y funciones de la dupla psicosocial, y; (ii) la tensión expresada entre las altas expectativas de la acción de la dupla y la baja importancia asignada a su trabajo (ver figura 2).

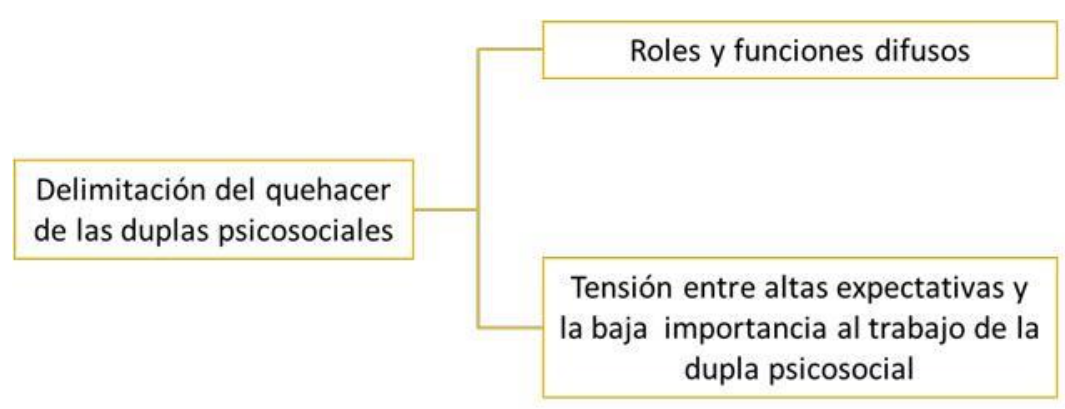

Figura 2. Dimensiones constitutivas del nudo crítico: delimitación del quehacer de las duplas psicosociales

\section{Poca claridad de los roles y funciones de la dupla psicosocial}

Respecto de la primera dimensión, podemos afirmar que uno de los elementos fundantes de la especificidad profesional es el rol esperado y el rol adscrito. En efecto, el rol confiere identidad y aparece como central cuando se trata de demarcar acciones profesionales. En este sentido, las narrativas recogidas señalan que un nudo primordial es la falta de claridad respecto de los roles y funciones desarrollados por la dupla psicosocial. Algunos de los relatos recogidos fueron:

“...en ocasiones se nos solicita acciones fuera de nuestro alcance..." (Grupo de Trabajo No3).

“...muchas veces se cuestiona el quehacer profesional de las duplas, debido al poco conocimiento de nuestro trabajo." (Grupo de Trabajo №) “...el psicólogo ve a los locos..." (Grupo de Trabajo No9).

El rol no es un elemento que se configura de manera independiente, al contrario, su construcción incorpora elementos contextuales, situacionales e históricos ineludibles. De este modo, distinguiremos tensiones desde el rol esperado, el rol percibido y el rol ejercido. El rol esperado hace alusión a las expectativas que los otros poseen acerca de las funciones a cumplir. En este caso, los relatos indican la existencia de una alta expectativa por parte de los profesores y directivos, en orden a que la actuación profesional de la dupla "normalice" la conducta de los estudiantes en un muy breve plazo, siendo recurrente la idea de la inmediatez del cambio y de la emergencia de resultados evidentes y permanentes en el tiempo.

El rol percibido se observa muy líquido (Bauman 2017). La narrativa indica que las duplas acomodan sus roles y funciones según las demandas de los agentes. Estos requerimientos muestran una alta variabilidad que transita desde atenciones individuales (centradas en lo cognitivo, la conducta disruptiva, las necesidades socioeconómicas); atenciones grupales (niños con NNE, convivencia escolar, bullying), derivaciones a redes de apoyo (programas de prevención focalizada, programas de reparación de maltrato, programas de intervención especializada); judicialización de los casos (medidas de protección). Desde esta líquida amplitud, emerge la sensación de roles y funciones imprecisas, que adoptan una alta variabilidad asociada a los requerimientos de cada escuela o comunidad educativa. Subyace a este respecto, un significado de rol de adaptación desmedida frente a las vicisitudes de cada contexto escolar y cultural, mediado por las exigencias distintas de cada centro, especialmente desde los cargos directivos. 
El rol ejercido corresponde a lo que realmente hacen las personas que ocupan determinada posición, bajo la consideración de elementos contextuales y situacionales. Como ya lo hemos mencionado, las duplas psicosociales relatan una enorme volubilidad en el rol ejercido, asociado a las distintas construcciones que de su rol hacen los agentes (directivos, profesores, apoderados) de su especificidad profesional. Esta ductilidad no es apreciada como positiva por los profesionales, al contrario, se muestran partidarios de trazar una delimitación de roles que ofrezca un espacio de desempeño laboral definido y que permita un ajuste de expectativas con miras a una descripción de cargo formal y estable.

\section{Tensión expresada entre las altas expectativas de la acción de la dupla y la baja importancia asignada a su trabajo}

La segunda dimensión está directamente vinculada con la primera, ya que a partir de las imprecisiones y vaguedades del rol y función de las duplas se advierte una tensión entre las altas expectativas hacia su acción, pero, a su vez, una sensación de baja importancia de su labor. Esta situación paradojal podría explicarse por algunas de las cuestiones ya señaladas en el nudo crítico de las condiciones laborales, y también desde la tensión entre la normalidad/anormalidad o la función/disfunción. El constructo simbólico instalado en esta dimensión se asocia a la inclusiónexcluyente que presiona al contexto escolar a aceptar lo diverso.

La aceptación de lo diverso, lo distinto, lo diferente, se instala a partir de una excesiva segmentación educativa que configura la existencia de guetos que no se intersectan. En efecto, la educación chilena ha configurado un sistema educativo segregado en clases sociales con la consecuencia indeseable de la excesiva homogeneidad dentro de cada segmento. Para el caso estudiado, la instalación de estas duplas se asocia textual y simbólicamente a las demandas de mayor inclusión. Pero ¿qué incluir?, la respuesta parece obvia, lo que ha estado excluido. Ergo, la pobreza, nuevos tipos de familia, conducta disruptiva, baja capacidad cognitiva, necesidades educativas especiales, vulnerabilidad social. De esta manera el "otro" a aceptar en las escuelas requiere de un dispositivo profesional encaminado a normalizar o a hacer funcional a aquel que aparece como distinto. Desde ahí, las expectativas son altas. Pero, todo aquel que trabaje con los diferentes o excluidos, termina por ser excluido también. Una de las formas de exclusión es la precariedad laboral, esto es, los que trabajan con aquellos sujetos que tienen precariedades sufren precariedades (O’Shea, Lysaght, Roberts \& Harwood 2016).

Así lo consigna uno de los relatos:

“...existe poca validación de las duplas psicosociales frente al grupo de docentes, desde el rol y ejercicio profesional". (Grupo de Trabajo N7)

\section{Intervención de la dupla psicosocial}

La intervención es una actividad dirigida a la solución de un problema práctico, pero que requiere de un marco conceptual y metodológico (Alvis 2009). A partir de este encuadre, en este nudo crítico se describen las principales dificultades a las que se enfrenta la dupla psicosocial, en el contexto de sus condiciones laborales y la difusa delimitación de su quehacer.

Las dimensiones construidas desde la narrativa son (i) Modelo de gestión de la convivencia en las escuelas; (ii) Estabilidad y continuidad de las intervenciones psicosociales; (iii) Trabajo en equipo y (iv) Derivación a redes externas (ver figura 3). 


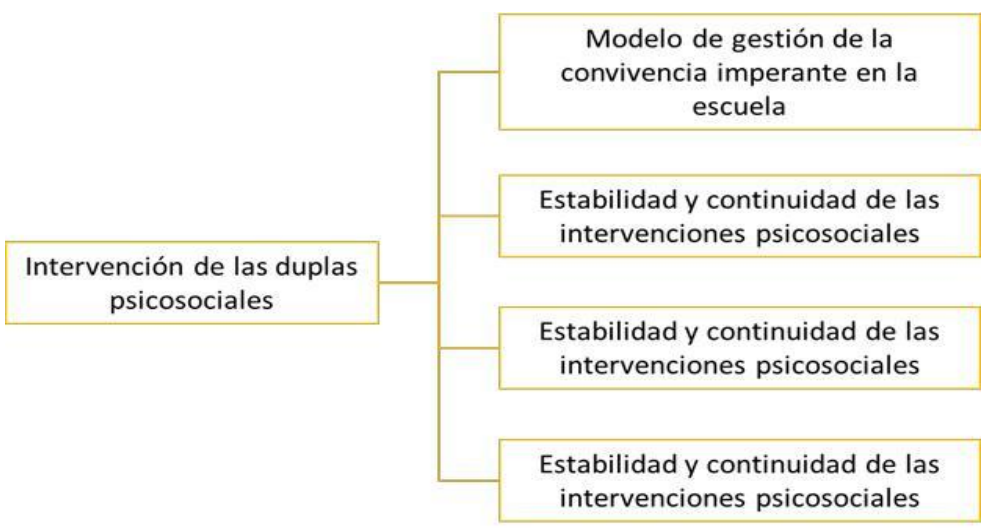

Figura 3. Dimensiones constitutivas del nudo crítico: intervención de las duplas psicosociales

\section{Modelo de gestión de la convivencia imperante en las escuelas}

Las duplas psicosociales relatan que el abordaje de la diferencia sigue colonizado por un discurso punitivo y su consecuente vigencia en la normativa. El modelo punitivo de la convivencia escolar se caracteriza por el énfasis en la normativa, desde una lógica de modelo redistributivo de la justicia (Torrego 2008, 2009); en este sentido, la hipótesis subyacente es que la aplicación de la sanción es la que disuade al infractor.

En este contexto, se genera una incongruencia entre el discurso oficial de inclusión y las prácticas cotidianas en los establecimientos educacionales respecto del uso habitual de la suspensión, aunque eufemísticamente se le llame reflexión al hogar. Desde lo simbólico, la diferencia sigue siendo tratada desde la exclusión del espacio escolar, ya sea sacando al estudiante de la sala de clases o apartándolo de la propia escuela, por unos días.

Desde la configuración de la intervención psicosocial, los usos de este tipo de medidas son contraproducentes con la construcción de un tipo de intervención que pretende estar enmarcada en el ámbito de los derechos humanos, con fuerte participación de los usuarios y que exige una contextualización imprescindible (Alvis 2009).

\section{Estabilidad y continuidad de las intervenciones psicosociales}

En cuanto a la estabilidad y continuidad de las intervenciones psicosociales, los discursos predominantes se refieren a la concomitancia entre la precariedad laboral de las duplas, lo que incluye inestabilidad de sus contratos y la posibilidad de establecer una relación profesional duradera y comprometida. En efecto, dentro de la intervención psicosocial se requiere que el profesional se configure como un actor comprometido y estable, para evitar victimización secundaria o baja adherencia a la intervención. En suma, las posibilidades de que la dupla psicosocial logre transformaciones en los individuos o en las familias requiere de un sistema de trabajo dónde predomine la personalización, la concientización y la creación de espacios de autonomía, todo lo cual, requiere condiciones laborales congruentes con este tipo de construcción.

\section{Trabajo en equipo}

Respecto del trabajo en equipo, esta dimensión se despliega discursivamente en la mejoría de la implicación, participación y compromiso de distintos actores. En primer lugar, se advierte que el espacio escolar aún es un campo de liderazgos autoritarios y ejercidos por profesores. Desde ahí, 
se observa una excesiva centralización del poder y un bajo acceso a la participación en la toma de decisiones, incluso en aquellas que son de responsabilidad de la dupla psicosocial. Esto genera tensiones frente a la disputa de quién es el experto en materias de intervención y quién es el que detenta formalmente el poder (equipo directivo).

En segundo lugar, las duplas observan una baja implicación y motivación de los profesores en lo que se refiere al trabajo desarrollado por los profesionales en su actuación psicosocial. Específicamente, predomina una conducta docente que, como lo mencionamos anteriormente, tiene altas expectativas respecto de la resolución rápida de la problemática del estudiante o de su familia, pero se margina de participar activamente. Ergo, no existiría un trabajo en equipo, entendido como la coordinación de coordinación de acciones (Maturana, 2008), sino más el bien el predominio de la racionalidad instrumental.

En tercer lugar, la construcción de equipo de trabajo también se ve disminuida por la baja adherencia y participación de las familias. Cabe precisar que esta participación se limita, habitualmente, a la madre o apoderada a cargo del niño o niña. En este sentido, se observa una resistencia a comprometer más tiempo en las intervenciones psicosociales que aquel que el habitus escolar ha logrado naturalizar (una reunión al mes, de tipo administrativo). Por otro lado, podría influir el dispositivo inclusión-exclusión, esto es, las madres que son llamadas a ser incluidas en una intervención psicosocial saben que su participación se origina en alguna causal de vulnerabilidad o de conductas disruptivas de sus hijos. En este sentido, investigación realizada indica que los/as apoderados muestran resistencia cuando se les llama para problematizar o patologizar la relación (Arón y Milicic 2000, 2004, Milicic y Arón 2000).

\section{Derivación a redes externas}

El trabajo en redes es una necesidad actual y creciente en el ámbito educativo. En efecto, la tendencia al aislamiento es un buen predictor de problemáticas psicosociales, ya sea en la esfera individual, familiar, grupal u organizacional. En este sentido, esta dimensión aparece como una cuestión a trabajar, desde la visión de las duplas, vinculado especialmente a la derivación a Tribunales de Familia o al Ministerio Público.

Cuando un niño/a sufre de una vulneración de sus derechos es obligación de las personas o de las instituciones denunciarlo a las instancias que correspondan. Habitualmente, esta acción tiende a ser rehuida por las consecuencias personales o laborales que puede acarrear. Es aquí donde se abre un flanco importante. Las duplas afirman que, como hay una gran variabilidad de tareas y un amplio espectro de funciones según el mandato institucional, se carece de un protocolo que establezca claramente quién es el responsable de denunciar, si lo hace en mérito de su cargo o como persona y cuáles son las reservas que una situación como esta amerita.

\section{Conclusiones}

Resulta evidente que, en la última década, las ideas de inclusión, equidad y género han alcanzado un nivel de aceptación mayoritario en el mundo de la educación. Consistentemente con este escenario, Chile ha introducido diversas reformas tendientes a la instalación de dispositivos proinclusión. En estas consideraciones finales, y parafraseando a Weinstein (2014), intentaremos develar la política de la política pro-inclusión que lleva a la instalación de las duplas psicosociales en las escuelas.

El abordaje del quehacer de las duplas psicosociales como objeto de estudio es reciente. La persistente inequidad de la que dan cuenta las pruebas estandarizadas a nivel nacional e internacional, sumado a la ubicuidad de la desigualdad educativa ponen de manifiesto la necesidad de problematizar respecto de esta temática. 
Posicionarse desde la perspectiva de los propios agentes que constituyen las duplas psicosociales presupone una apuesta por el desarrollo de una línea investigativa de gran proyección. La sola existencia de diversos instrumentos de gestión y planificación dirigidos a la mejora de la calidad educativa y la reducción de la brecha existente entre los diversos establecimientos educacionales presentes en el territorio nacional no bastan. Es crucial atender a los agentes que intervienen de forma situada en cada uno de los contextos en los cuales son requeridos. Desde esta perspectiva, el análisis de lo informado por los grupos de trabajo nos permite destacar la existencia de tres nudos críticos cuya articulación en función de círculos concéntricos facilitan la representación de la alta complejidad del fenómeno.

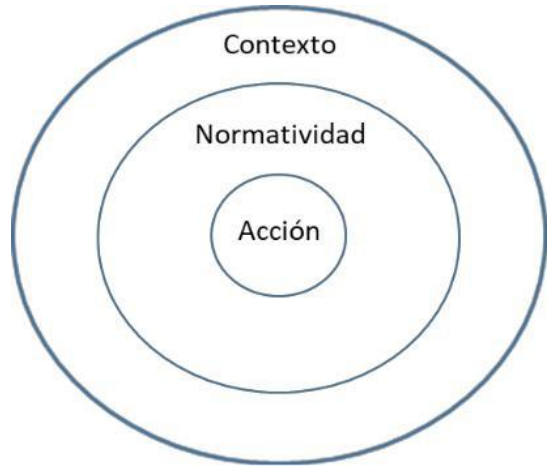

Figura 4. Dimensiones constitutivas de la dupla psicosocial como fenómeno

Dada la recursividad de las dimensiones constitutivas, las consideraciones finales que se exponen a continuación deben ser decodificadas desde la lógica de la interdependencia que subyace al actuar de las duplas psicosociales dentro del sistema escolar. De este modo, contexto, normatividad y acción se manifiestan como una totalidad donde el análisis no considera jerarquía o causalidad lineal; los elementos constitutivos del fenómeno se nos presentan imbricados holísticamente. Sin embargo, para efectos de una mejor comprensión se entenderá como contexto aquellos elementos que configuran las condiciones estructurales y administrativas donde se instala el dispositivo de la dupla psicosocial. En tanto, la normatividad se corresponde con el entramado reglamentario y simbólico a partir del cual surgen las operaciones de distinción respecto de sus roles y funciones. Finalmente, la acción alude a la manifestación empírica de la intervención psicosocial de las duplas.

Sobre la base de lo expuesto, los actores principales que definen la política pro-inclusión serían, por una parte, las duplas psicosociales, en tanto equipo profesional de reciente inserción en las escuelas y los equipos directivos de las escuelas. Entre ambos actores existen diferencias notables. El equipo directivo detenta el poder de decisión; se les atribuye una importancia no sólo simbólica, sino que práctica en el quehacer de las comunidades educativas; han configurado un saber experto expresado en un habitus reconocible y funcionan como sistemas autopoiéticos frente a las transformaciones o perturbaciones del sistema escolar. Las duplas psicosociales subsisten afrontando la liquidez de su delimitación de funciones; experimentan precariedad laboral que impacta directamente en su labor; están configurando recientemente un saber experto en materia técnica de intervención psicosocial; este saber experto no recibe la validación y legitimación por parte de los decisores.

A estos actores principales, cabría agregar algunos actores influyentes en la configuración de esta política. Nos referimos a los docentes y a las madres, padres y apoderados. Estos agentes registran un rol demandante/receptor, esto es, pueden activar requerimientos hacia la dupla psicosocial en el contexto de la normalización o logro de la funcionalidad de aquellos definidos como fuera de norma o disfuncionales. Asimismo, pueden ser seleccionados o elegidos como receptores de una intervención; en este caso, tanto profesores como apoderadas muestran 
resistencias a la participación activa, eludiendo enérgicamente, pero desde distinto lugar, la adherencia a esta intervención. En el caso de los profesores su baja adherencia se sitúa desde la lógica de que su rol es otro, esto es, educar. Para las apoderadas, su negativa se sitúa en la resistencia a ser patologizadas o etiquetadas como problemáticas.

Respecto de los procesos desarrollados para la instalación de las duplas psicosociales, se advierte una diversidad de inconvenientes vinculados a la precariedad laboral, esto es, inestabilidad de los contratos, diferentes fuentes de financiamiento (SEP, PIE), carencia de espacio físico para el desarrollo de su labor, todos los cuales resultan incongruentes con la continuidad de la intervención psicosocial y con los principios de confidencialidad y personalización. Otras contrariedades aparecen en el marco de la delimitación de las funciones de la dupla, definición que registra alta variabilidad según el tipo de contrato y los requerimientos específicos de cada establecimiento educacional. Finalmente, se observa insuficiencia en la construcción social de un proceso de inclusión educativa que se sitúe como un eje central de la labor de las escuelas. En esta construcción colectiva debería ganar fuerza el trabajo en equipo, las decisiones basadas en criterios, la validación del trabajo experto de la dupla, la generación de protocolos de atención a las graves vulneraciones de derechos de niños, niñas y adolescentes, así como una mayor clarificación de la necesidad, pertinencia y utilidad de la existencia de las duplas psicosociales como un elemento constituyente de la sinergia sistémica de la comunidad educativa.

Finalmente, se distinguen tensiones entre lo político y lo técnico, como una expresión del nuevo management público y de la vigencia de un modelo centrado en las evidencias que presiona, excesivamente, la rendición de cuentas. Este modelo no garantiza que la mera materialización de la política de inclusión logre la consolidación de un sistema educativo inclusivo. Desde esta perspectiva, no basta con instalar duplas psicosociales en las escuelas para que se configure, desde un imaginario casi mágico, un nuevo nicho ecológico que permita, de manera genuina, una disposición a que "el otro" aparezca en su legitimidad como ser humano (Maturana, 2008). Lograr una auténtica inclusión educativa supone que la comunidad educativa, en tanto sistema autopoiético, se deslice más allá que lo que ha hecho hasta ahora y aplique cambios de segundo orden, en el sentido de permitir que el sistema se mire a sí mismo y ofrezca las condiciones para cuestionar su conservación tal cual está. En este sentido, se observa aún un predominio de lo que denominamos clausura disciplinar pedagógica, entendida como el proceso dirigido a mantener la homeostasis del sistema, impidiendo o dificultando el ingreso de todo aquello que se represente como ajeno o exterior a la función principal de la escuela: la enseñanza.

Probablemente, una de las claves para destrabar los procesos de morfostásis educativa es la transformación global de la convivencia escolar. Un obstáculo para lograrlo es que aún la educación chilena no se despoja de las mediciones estandarizadas del aprendizaje, dónde la convivencia no aparece sino como un mero apéndice. Ergo, hasta que no avancemos hacia una intervención ortogonal educativa, las duplas psicosociales podrían seguir siendo vistas sólo como instrumentales y, en consecuencia, quedando relegadas al territorio de lo periférico, de lo que está en el margen. A nuestro juicio, lo que debería estar en el centro es la construcción de una convivencia humana que mejore los aprendizajes, y recursivamente, los logros de aprendizaje nutrirán una buena convivencia.

\section{Referencias}

Alvis, A. (2009). Aproximación teórica a la intervención psicosocial. Poiésis, 17, 1-6. Apablaza, M. (2014). Representaciones sociales de profesores respecto de la diversidad escolar en relación a los contextos de desempeño profesional, prácticas y formación inicial. Estudios Pedagógicos, XI(1), 7-24.

Apablaza, M. (2016). Discursos de Diferencia en Educación: Análisis de la Política de Diversidad/Inclusión Chile. E Investigação Qualitativa em Educação (Vol. 1, pp. 336345). Portugal.

Apablaza, M. (2017a). Prácticas "psi” en el espacio escolar: Nuevas formas de subjetivación de 
las diferencias. Psicoperspectivas. Individuo y Sociedad, 16(3). Recuperado de https://doi.org/10.5027/psicoperspectivas-Vol16-Issue3-fulltext-1063

Apablaza, M. (2017b). Producción de las diferencias en la escuela: Análisis de la política de inclusión educativa chilena. Presentado en Cuarto Congreso Interdisciplinario de Investigación en Educación, Santiago de Chile.

Arón, A., \& Milicic, N. (2004). Clima social escolar y desarrollo personal: un programa de mejoramiento. Santiago: Andrés Bello.

Arón, A., \& Milicic, N. (2000). Desgaste profesional de los profesores y clima escolar. Revista Latinoamericana de Psicología, 32, 3, 447-466.

Bauman, Z. (2017). Modernidad Líquida. Madrid: Fondo de Cultura Económica.

Bellei, C. (2013). Situación Educativa de América Latina y el Caribe: Hacia la educación de calidad para todos al 2015. ORELAC, UNESCO. Santiago, Chile.

Bourdieu, P., \& Passeron, J. (1979). La reproducción. Elementos para una teoría del sistema de enseñana. Barcelona: Laia.

Chacón Reyes, J. (2007). “Educación, interdisciplinariedad y pedagogía”. Revista Pampedia N³. Universidad de Veracruz, México.

Du Ranquet, M. (1996). Los modelos en trabajo social: intervención con personas y familias. Madrid: Siglo Veintiuno Editores.

Ezcurra, A. M. (2011). Igualdad en Educación Superior. Un desafío mundial. Los Polvorines: Universidad Nacional de General Sarmiento; IEC-CONADU.

Ferrada, D. (2012). Construyendo escuela, compartiendo esperanzas. La experiencia del proyecto "Enlazando Mundos". Santiago: RIL.

Ffrench-Davis, R. (1999). Entre el neoliberalismo y el crecimiento con equidad. Tres décadas de política económica en Chile. Santiago: Dolmen.

Garreta, J. (2015). La Comunicación Familia-Escuela en Educación Infantil y Primaria. Revista de la Asociación de Sociología de la Educación, 8,1.

Gatica, F. (2015). Las intervenciones psicosociales en establecimientos educacionales municipales bajo el marco de la Ley SEP. Diseño, implementación y logros desde la perspectiva de actores claves (Tesis de Maestría). Facultad de Ciencias Físicas y Matemáticas, Departamento de Ingeniería Industrial. Universidad de Chile. Santiago. http://repositorio.uchile.cl/handle/2250/133241

Gatica, F. (2016). Las intervenciones psicosociales en establecimientos educacionales municipales vulnerables bajo el marco de la Ley SEP. Revista Estudios de Políticas Públicas, 3(2), 105-119

Gatica, N., \& Hess, V. (2011). Construcción Social de la Intervención/Actuación Psicosocial desde el discurso de trabajadores/as sociales y psicólogos/as en las áreas de salud e infancia. Tesis para obtener Título de Trabajadora Social. Universidad del Bío-Bío, Chillán, Chile.

Giraldo-Zuluaga, G. A. (2015). Ciudadanía: aprendizaje de una forma de vida. Educación y Educadores, 18(1), 76-92. https://doi.org/10.5294/edu.2015.18.1.5

González, M., González, I., \& Vicencio, K. (2014). Descripción del rol autopercibido del psicólogo y sus implicancias en los procesos de formación en pregrado. Psicoperspectivas. Individuo y Sociedad, 13(1). doi: https://doi.org/10.5027/psicoperspectivas-Vol13-Issue1-fulltext-296

Gubbins, V. (2016). Relación familias y escuelas: ¿por qué y para qué? Santiago: Universidad Finis Terrae.

Infante, M. D., \& Matus, C. (2009). Policies and practices of diversity: reimagining possibilities for new discourses. Disability \& Society, 24(4), 437-445. https://doi.org/10.1080/09687590902879049

Junta Nacional de Auxilio Escolar y Becas (2005). SINAE. Sistema Nacional de Asignación con Equidad para Becas JUNAEB. Una nueva visión en la construcción de igualdad de oportunidades en la infancia. [Versión electrónica http://www.junaeb.cl]. Santiago: Junta Nacional de Auxilio Escolar y Becas-Chile.

Maturana, H. (2008). El sentido de lo humano. Buenos Aires: Granica.

Maturana, H. (1996). La realidad: ¿objetiva o construida? Guadalajara: Anthropos. 
Matus, C., \& Infante, M. (2011). Undoing diversity: knowledge and neoliberal discourses in colleges of education. Discourse: Studies in the Cultural Politics of Education, 32(3), 293-307. doi: https://doi.org/10.1080/01596306.2011.573248

Milicic, N.; Arón, A. (2000). Climas sociales tóxicos y climas sociales nutritivos para el desarrollo personal en el contexto escolar. Psykhe, 9(2), 117-123.

Muñoz, J. M. E., González, M. T. G., \& Domínguez, B. M. (2009). El fracaso escolar como exclusión educativa: comprensión, políticas y prácticas. Revista iberoamericana de educación, 50, 41-64.

Programa de Naciones Unidas para el Desarrollo (PNUD) (2017). Des-iguales: orígenes, cambios $y$ desafios de la brecha social en Chile. Santiago: PNUD-UQBAR

Raczynsky, D.; Muñoz, G.; Weinstein, J.; Pascual, J. (2013). Subvención escolar preferencial (SEP) en Chile: un intento por equilibrar la macro y micro política escolar. REICE. Revista Iberoamericana sobre Calidad, Eficacia y Cambio en Educación, 11, 2, 164-193.

Raczynski, D., \& Muñoz, G. (2007). Reforma educacional chilena: el difícil equilibrio entre la macro y la micropolítica. REICE. Revista Iberoamericana sobre Calidad, Eficacia y Cambio en Educación, 5, 3, 40-83.

Rockwell, E. (2009). La experiencia etnográfica. Historia y cultura en los procesos educativos. Buenos Aires: Paidós.

Salazar, G., \& Pinto, J. (1999). Historia contemporánea de Chile I: Estado, legitimidad, ciudadanía. Santiago: LOM.

Saracostti, M. (2013). Familia-escuela comunidad I: Una alianza necesaria para un modelo de intervención biopsicosocial positivo. Santiago: Editorial Universitaria.

Trinidad, A., Carrero, V., \& Soriano, R. (2006) Teoría fundamentada. "Grounded Theory". La construcción e la teoría a través del análisis interpretacional. Madrid: CIS.

Torche, F., \& Wormald, G. (2004). Estratificación y movilidad social en Chile: entre la adscripción y el logro. Santiago: CEPAL.

Torrego, J. (2009). Modelo integrado de mejora de la convivencia: estrategias de mediación y tratamiento de conflictos. Barcelona: Graó.

Torrego, J. (2008). Mediación y resolución de conflictos en instituciones educativas. Santiago: Fundación Creando Futuro.

Valenzuela, J.P., Bellei C., \& De los Ríos, D. (2010). Segregación escolar en Chile. En Martinic, S. \& Elacqua, G. (eds.) Fin de Ciclo: Cambios en la Gobernanza del Sistema Educativo. Santiago, Facultad de Educación, Pontificia Universidad Católica de Chile y Oficina Regional para América Latina y el Caribe UNESCO, pp. 209-229.

Weinstein, J. (2014). La esquiva política entre las volátiles políticas docentes. En OREALCUNESCO, Temas críticos para la elaboración de políticas docentes en América Latina y El Caribe. P. 188-227. Santiago: OREALC-UNESCO

Yeager, D., Walton, G. (2011). Social-Psychological Interventions in Education: They're not magic. Review of Educational Research. Stanford University Libraries.

\section{Financiamiento:}

Este trabajo fue patrocinado por el Grupo de Investigación Familia, Escuela y Sociedad FESOC (GI 170124/EF), Dirección de Investigación Universidad del Bío-Bío y el proyecto de investigación Fondecyt $\mathrm{N}^{\circ} 11160084$ de CONICYT 